\title{
ENERGY RECOVERY TRANSPORT DESIGN FOR PKU FEL"
}

\author{
Guimei Wang", Yu-Chiu Chao, Chuyu Liu, Jefferson Lab, Newport News, VA, USA \\ Guimei Wang, Kui Zhao, Xiangyang Lu, Jiejia Zhuang, Chuyu Liu, Zhenchao Liu, Jiaer Chen, \\ Institute of Heavy Ion Physics \& MOE Laboratory of Heavy Ion Physics, Peking University, \\ Beijing, China
}

\section{Abstract}

A SRF linac based free electron laser user facility is under developed at Peking University. Energy recovery Linac technology was chosen for increase of average electron beam current, hence, increase of the free electron laser power. In this paper we present a conceptual design of beam transport line which satisfies requirement of ERL. A chicane consisting of four identical bend magnets is selected for path length adjustment up to \pm 18 degree. R56 of both arcs of the beam line is adjustable for full bunch compression.

\section{INTRODUCTION}

Peking University is building a free-electron laser facility for experimental studies on nonlinear transient physical processes, chemical kinetics, molecular biology, material science and so on. It will run in the IR $(5 \sim 10 \mu \mathrm{m})$ and $\mathrm{THz}$ region.

The facility is basically composed of a $3+1 / 2$ DC-SC photo injector [1], a $1.3 \mathrm{GHz}$ superconducting accelerator, a bunch compressor, and a wiggler. Due to the superconducting characteristics of the injector and accelerator, this facility has the ability to provide $\mathrm{CW}$ electron beams. With the pioneering ERL FEL successful experimental results in mind from the Jefferson Lab FEL, the JAEA FEL and the Novosibirsk FEL, the feature of energy recovery technology, maintaining high average and peak brightness of the electron beam, makes energy recovery very attractive not only in an FEL, but also when applied in a synchrotron light source. Now, energy recovery technology is chosen as the most promising way to increase FEL power in PKU.

In this paper, the design of the beam transport system for energy recovery is presented.

\section{SYSTEM REQUIREMENTS}

The beam transport line for energy recovery, firstly, is to deliver the electron beam to the wiggler with properties fulfilling FEL requirements, including the beam emittance, the energy spread and the beam current. It should also return the electron beam with large energy spread after FEL interaction to the accelerator at a certain deceleration phase, converting the electrons' energy to RF power at high efficiency. So it requires the beam line after the wiggler to the accelerator to have large momentum acceptance.

\footnotetext{
* Work supported by National 973 Projects and U.S. DOE Contract No. DE-AC05-06OR23177

\#wangguimei@pku.edu.cn, +chao@jlab.org
}

The detailed information for the $3+1 / 2$ DC-SC photo injector, running at $1.3 \mathrm{GHz}$, is described in reference [1]. Simulation shows that it has the potential to provide beam with bunch charge of $100 \mathrm{pC}$, transverse normalized emittance lower than 2 mm.mrad after solenoid compensation and bunch length 6 ps (FWHM). The 1.3 $\mathrm{GHz}$ superconducting accelerator can accelerate an electron beam to about $25 \mathrm{MeV}$. As mentioned above, the FEL will provide light in the IR region. The wiggler and required electron beam parameters are listed in table 1 .

Table 1 Beam and Wiggler Parameters

\begin{tabular}{|l|l|}
\hline \multicolumn{2}{|c|}{ Wiggler } \\
\hline Period of Wiggler & $2.7 \mathrm{~cm}$ \\
\hline Number of Wiggler Period & 60 \\
\hline K_rms & $\sim 0.7$ \\
\hline Raylength & $0.6 \mathrm{~m}$ \\
\hline Wavelength of FEL & $5 \sim 10 \mu \mathrm{m}$ \\
\hline \multicolumn{2}{|c|}{ Electron Beam Parameters } \\
\hline RF Frequency & $1.3 \mathrm{GHz}$ \\
\hline Beam Rep. Rate & $26 \mathrm{MHz}$ \\
\hline Inject Energy & $5 \mathrm{MeV}$ \\
\hline Energy Gain in Accelerator & $25 \mathrm{MeV}$ \\
\hline RMS Energy Spread & $0.4 \%$ \\
\hline Bunch Charge & $>20 \mathrm{pC}$ \\
\hline Transverse normalized Emittance & $<20 \mathrm{~mm}-\mathrm{mrad}$ \\
\hline Beta X at wiggler & $1.7 \mathrm{~m}$ \\
\hline Alpha X at wiggler & 1.35 \\
\hline Beta Y at wiggler & $0.37 \mathrm{~m}$ \\
\hline Alpha Y at wiggler & 0 \\
\hline
\end{tabular}

The FEL power is proportional to the beam peak current, so it is better to get as short bunch length as possible. The common way to get a short bunch is magnetic compression and RF compression. Here, the magnetic compression is chosen, which requires energy-position correlation. So the accelerator phase of the electron beam should provide this correlation, while fulfilling the energy spread requirements from the FEL.

To recover the electron beam energy after the FEL, the recirculated beam should be returned to the accelerator at a certain phase with respect to the RF fields. This can be carried out through path length adjustment. After the FEL, the energy spread of the electron beam is very large and the bunch length is very short. The energy spread after deceleration should be small to transport the low energy beam to the dump. So the beam transport after the wiggler to the dump should have energy spread compression [2]. 
The footprint for the facility is $23 \mathrm{~m}$ long and $7 \mathrm{~m}$ wide. The transport system should fit it.

\section{PATH LENGTH ADJUSTMENT}

In the recirculation, the electron speed is almost the same as the speed of light. To change the path length, the common way is to change the beam trajectory in the magnet or mechanically move the arc. Here four rectangle bend magnets composing the chicane structure as shown in Fig. 1 are selected to change path length.

The magnet projection length is $l_{\text {eff }}$, the bend radius is $r$ and the bend angle is $\alpha$. Inside the magnet, the trajectory length is $l_{a r c}$ and $x_{a r c}$ is the horizontal displacement. The projection length between the first magnet boundary and the second magnet boundary is $d, l_{d}$ is the drift space from the first magnet to the second one, and $x_{d}$ is the horizontal displacement in the drift space.

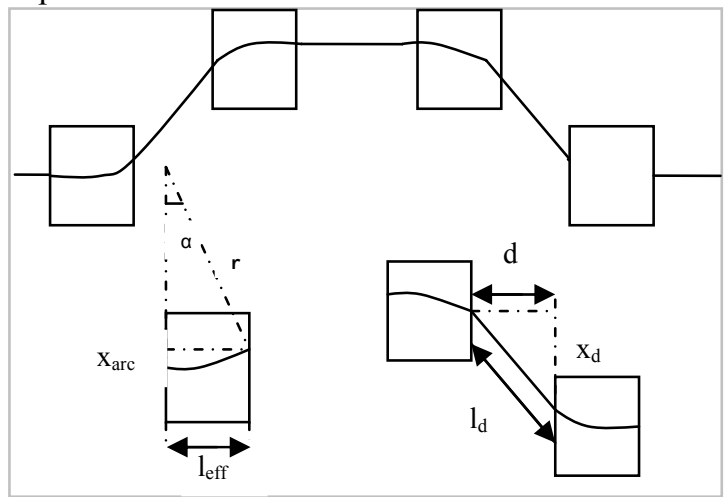

Figure 1: chicane structure

From the geometry in Fig.1, we have these relations

$$
\begin{aligned}
& l_{\text {eff }}=r \sin \alpha \\
& l_{\text {arc }}=l_{\text {eff }} \cdot \frac{\alpha}{\sin \alpha} \\
& l_{d}=\frac{d}{\cos \alpha}
\end{aligned}
$$

The path length difference between the chicane and the projection is

$$
\begin{aligned}
& \Delta l=4\left(l_{\text {arc }}-l_{\text {eff }}\right)+2\left(l_{d}-d\right) \\
& =4 l_{\text {eff }}\left(\frac{\alpha}{\sin \alpha}-\alpha\right)+2 d\left(\frac{1}{\cos \alpha}-1\right)
\end{aligned}
$$

So, changing bend angle $\alpha$, the path length will change.

The horizontal displacement

$$
x=2 l_{\text {eff }} \frac{(1-\cos \alpha)}{\sin \alpha}+d \tan \alpha
$$

And $R_{56}$ will also change with $\alpha$.
Table 2 Bend Angle Scan for Chicane

\begin{tabular}{|c|c|c|c|c|c|}
\hline $\begin{array}{c}\text { Bend } \\
\text { angle }(\text { degree })\end{array}$ & 19 & 19.5 & 20 & 20.5 & 21 \\
\hline$\delta l(\mathrm{~cm})$ & -2.29 & -1.16 & 0 & 1.2 & 2.43 \\
\hline$\delta \psi_{r f}($ degrees $)$ & -35.7 & -18.1 & 0 & 18.74 & 37.97 \\
\hline$\delta x(\mathrm{~cm})$ & -3.67 & -1.84 & 0 & 1.847 & 3.71 \\
\hline$R_{56}(\mathrm{~cm})$ & -43.8 & -46.5 & -49.3 & -52.1 & -55.1 \\
\hline$R_{16}(\mathrm{~cm})$ & 71.9 & 74.4 & 76.8 & 79.37 & 81.9 \\
\hline
\end{tabular}

After optimization, $l_{\text {eff }}$ is set to be $0.4 \mathrm{~m}, d$ is $1.5 \mathrm{~m}$, and $\alpha$ is 20 degrees, changing \pm 0.5 degree. Table 2 shows the $\delta l$, rf phase change $\delta \psi_{r f}$ and $\delta x$, which correspond to a 20 degree shift, $R_{56}$ and maximum $R_{16}$. This chicane is set before the wiggler, which is good for beam size control at small energy spread, providing bunch compression and path length adjustment. From this table, we can see that the beam size contributed from dispersion is about $2 \mathrm{~cm}$ at $6 \sigma_{\delta p / p}$, but the displacement at 19 or 21 degrees can reach $3.7 \mathrm{~cm}$. To constraint the beam pipe to a reasonable size, the bend angle will change from 19.5 degrees to 20.5 degree. It can give 18 degrees RF phase change.

\section{ARC DESIGN}

As mentioned above, the beam should accelerate at a certain phase to provide energy-position correlation to get short bunch length and maintain energy spread under FEL requirements.

Setting the beam in the phase off crest $\varphi_{r f}$, we can get energy position correlation and the FWHM energy spread $\left(\frac{\Delta E}{E}\right)^{F E L}:\left(\frac{\Delta E}{E}\right)^{F E L}=2 \frac{E_{F E L}-E_{i n j}}{E_{F E L}} \tan \varphi_{r f} \sin \left(\frac{\pi L_{b u n c h}^{i n j}}{\lambda_{r f}}\right)$ where $E_{F E L}$ is the electron energy at the wiggler, $E_{i n j}$ is the electron energy after the injector, $\lambda_{r f}$ is the wavelength of RF and $L_{b u n c h}^{i n j}$ is the FWHM bunch length after the injector.

From reference [2], to get the shortest bunch length or highest beam current, $R_{56}$ should be

$R_{56}=\frac{\lambda_{r f}}{2 \pi \tan \varphi_{r f}} \frac{E_{F E L}}{E_{F E L}-E_{i n j}}$, i.e. $-19 \mathrm{~m}$, which corresponds to an accelerator phase of -13 degrees. With the chicane changing the path length from -18 degrees to 18 degrees, $R_{56}$ will change, which will be compensated by the arc before the wiggler, from $27 \mathrm{~cm}$ to $33 \mathrm{~cm}$. The arc after the wiggler should give $R_{56} 19 \mathrm{~cm}$ to compress energy spread and be capable to transport large momentum beam without loss. 
In the design, the arc before and after the wiggler has the same structure. As shown in Fig.2, it is a symmetric structure, using four identical rectangle bends, each with a

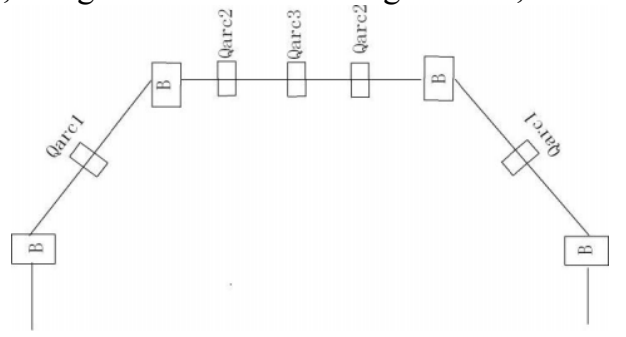

Figure 2: layout of arc

45 degree bend angle, and three quads to adjust $R_{56}$, achromats and also the beam envelope. In this structure, $R_{56}$ is uniquely controlled by the quadrupole Qarc1, and quads Qarc2 and Qarc3 can balance each other to get achromatic.

The field of bends is $2.03 \mathrm{kG}$, bend radius is $0.5 \mathrm{~m}$, bend length is $0.3927 \mathrm{~m}$ and the arc length is $5.82 \mathrm{~m}$, with the $4.26 \mathrm{~m}$ width and $1.4 \mathrm{~m}$ length. Fig. 3 shows the beam envelope with the same initial twiss parameters for different $R_{56}, 19 \mathrm{~cm}, 27 \mathrm{~cm}$ and $33 \mathrm{~cm}$. The strength of Qarc1 changes by a small amount, from $0.2 \mathrm{kG} / \mathrm{cm}$ to $0.22 \mathrm{kG} / \mathrm{cm}$. It shows that the beam envelope goes through arc consistently without large oscillation caused by change in quad strength. The largest beta is $20 \mathrm{~m}$, in the vertical direction and $5 \mathrm{~m}$ in the horizontal direction, which gives a very small contribution to the beam size, and the largest $R_{16}$ is $0.53 \mathrm{~m}$.

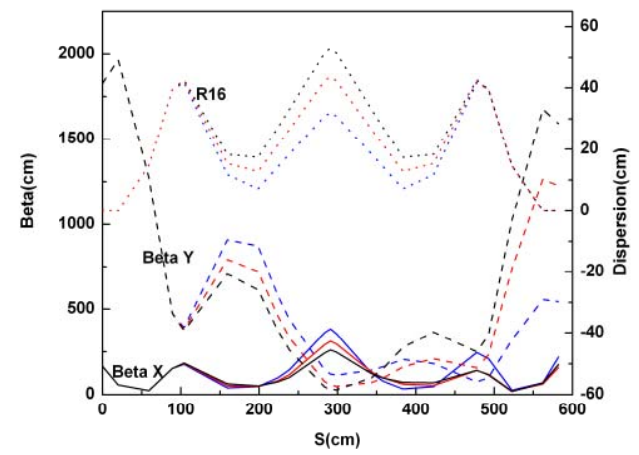

Figure 3: Beta and dispersion propagation along the arc for different $R_{56}$ (Blue: $19 \mathrm{~cm}$, red: $27 \mathrm{~cm}$, black: $33 \mathrm{~cm}$ )

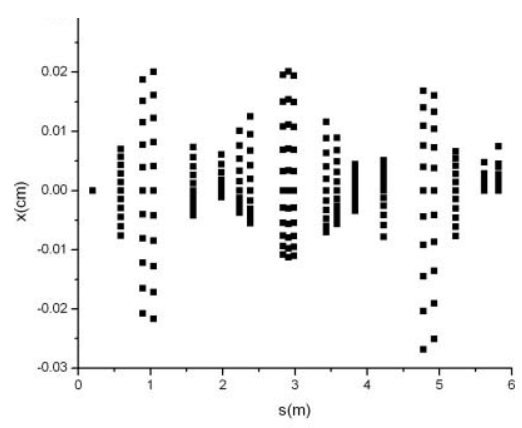

Figure 4: Beam orbit along the arc for different energy spread from $-5 \%$ to $5 \%$
Fig.4 is the central orbit along the arc for different energy spreads scanned up to $5 \%$, using the code Dimad. The largest beam size is up to $3 \mathrm{~cm}$.

\section{OPTICS FOR THE RECIRCULATION}

The beam line connecting the accelerator to the first arc is four quads to match the beam to arc1, and the common chicane structure is as stated above in Fig.1, which separates the accelerated beam to arc1 and the decelerated beam to dump, with a 3.7 degree bending angle for $30 \mathrm{MeV}$ and 20 degree for $5 \mathrm{MeV}$. For the transport from the second arc to the accelerator, it is similar as above; the chicane is to combine injection beam and recirculation beam to the accelerator.

The circumference of the beam line is $40.03846 \mathrm{~m}$, the length is $16.9 \mathrm{~m}$ and the width for the recirculation is 4.26 m. Fig. 5 shows the beta function and dispersion along the recirculation. The largest dispersion is at the chicane before the wiggler, where the energy spread is small. In the second arc, although the energy spread is large, the dispersion is about $0.4 \mathrm{~m}$ and beta $\mathrm{X}$ is small, about $5 \mathrm{~m}$, so it can transport large momentum spread beam.

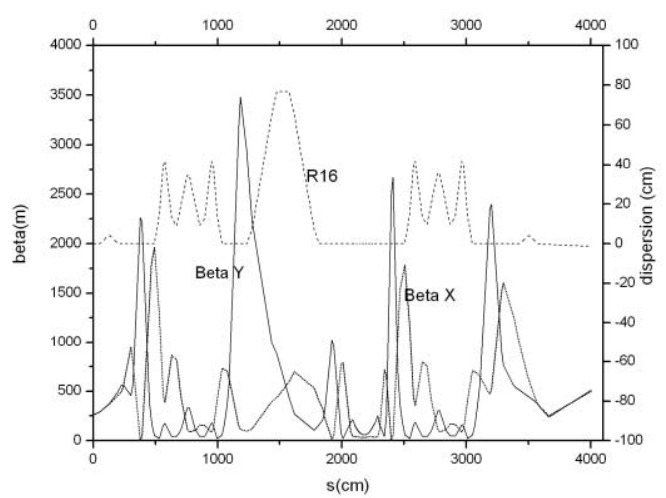

Fig.5 Beam optics along the recirculation

\section{SUMMARY}

The energy recovery transport line for Peking University is presented. The chicane before the wiggler can adjust path length from -18 degrees to +18 degrees rf phase, resulting in $R_{56}$ changes, which can be compensated by the first arc. In the arc, $R_{56}$ is uniquely controlled by the Qarc1. And dispersion in the second arc is about $0.4 \mathrm{~m}$. The line has the ability to transport large energy spread beam.

\section{REFERENCES}

[1] Zhu Feng et al., Updated design of the DC-SC photoinjector at Peking University, High power laser and particle beams, 2006(18):653-656

[2] P.Piot et al., Physical Review Special TopicsAccelerators and Beams, 2003(6):030702 